\title{
Metabolic syndrome and insulin resistance: Can they be predicted by clinical indicators in obese prepubertal children?
}

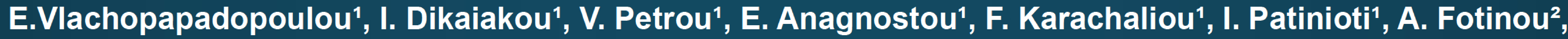 S. Michalacos ${ }^{1}$}

1.Dept. of Endocrinology-Growth and Development, Children's Hospital P. \& A. Kyriakou, Athens, Greece 2. Biochemistry Dept.-Hormones Laboratory, Children's Hospital P. \& A. Kyriakou, Athens, Greece

\section{AIM}

To evaluate whether anthropometric indices and acanthosis nigricans can be useful markers for early detection of Insulin Resistance and Metabolic Syndrome (MetS) in overweight and obese children

\section{METHODS}

Data from 510 prepubertal children ( $40 \%$ boys), $12.9 \%$ overweight and $87.1 \%$ obese with mean age $9.7 \pm 2.5$ years were analyzed. Logistic regression analysis was used to investigate which factors were associated with HOMA-IR $>3$ and metabolic syndrome.

\section{RESULTS}

- MetS was found in $12.9 \%$ of the children.

- HOMA-IR $>3$, was found in $14.3 \%$ of overweight and $39.8 \%$ of obese children

- Among children with MetS, 50\% had HOMA-IR $>3$ and they were all obese.

- The mean Body Mass Index (BMI) was greater in children with HOMA-IR >3 (29.3 \pm 3.1 vs. 26.1 $\pm 3.1, p<0.001)$ (OR:1.31, 1.20-1.44).

- Also, children with HOMA-IR $>3$ had greater waist circumference (mean \pm SD: $94.7 \pm 9.6$ vs. $85.6 \pm 10.4, p<0.001$ ).

- Acanthosis Nigricans (OR=2.42, 95\% Cl: $1.23-4.79, p=0.011$ ) and increased $\%$ fat $(\mathrm{OR}=1.14,95 \% \mathrm{Cl}: 1.05-1.25, \mathrm{p}=0.003)$ were associated with greater likelihood for HOMA-IR $>3$.

- Waist-to-height (WHtR) was associated with greater odds for HOMA-IR > 3 (OR=1.07, 95\% Cl: $1.02-1.18, p=0.013)$.

- Increased WHtR tended to be associated with the presence of MetS (OR=1.07, 95\% Cl: $0.99-1.16, p=0.100)$, while for one unit increase in BMI the likelihood for MetS was found to increase about $16 \%$ $(p=0.001)$.

\section{CONCLUSION}

The severity of obesity as evidenced by BMI, the presence of waist circumference to height ratio higher than 0.5 , as well as the presence of acanthosis nigricans are clinical indicators of increased metabolic risk.

Children at increased risk should be followed closely and have a more intense program of healthy diet and increased physical activity.

\begin{tabular}{|l|l|c|c|}
\hline \multicolumn{2}{|c|}{ Age(SD) } & N & $\%$ \\
\hline \multirow{2}{*}{ Gender } & Males & $9.7(2.5)$ & \\
\cline { 2 - 4 } & Females & 204 & 40.0 \\
\hline \multirow{2}{*}{ BMI (kg/m²) (SD) } & 306 & 60.0 \\
\hline \multirow{2}{*}{ BMI (kg/m²) } & Overweight & $26.1(4.1)$ & \\
\cline { 2 - 4 } & Obese & 64 & 12.9 \\
\hline \multirow{2}{*}{ WtH ratio, (SD) } & 433 & 87.1 \\
\hline \multirow{2}{*}{ \% Fat, (SD) } & & $0.61(0.05)$ & \\
\hline \multirow{2}{*}{ HOMA-IR >3 } & $<3$ & $35.9(5.7)$ & \\
\cline { 2 - 4 } & $>3$ & 142 & 60.9 \\
\hline \multirow{2}{*}{ Metabolic syndrome } & 0 & 91 & 39.1 \\
\cline { 2 - 4 } & 1 & 243 & 87.1 \\
\hline \multirow{2}{*}{ W. C. (SD) } & & 36 & 12.9 \\
\hline \multirow{2}{*}{ Positive hy for obesity } & NO & $28.2(11.0)$ & \\
\cline { 2 - 4 } & YES & 239 & 48.4 \\
\hline Acanthosis nigricans & NO & 438 & 81.6 \\
\cline { 2 - 4 } & YES & 63 & 12.6 \\
\hline
\end{tabular}

\begin{tabular}{|c|c|c|c|c|c|c|c|}
\hline & \multicolumn{4}{|c|}{ HOMA-IR } & \multirow{3}{*}{$\begin{array}{l}\text { OR } \\
(95 \% \mathrm{Cl})\end{array}$} & \multirow{3}{*}{$\mathbf{P}$} \\
\hline & & \multicolumn{2}{|c|}{$<3$} & \multicolumn{2}{|c|}{$>3$} & & \\
\hline & & $\mathrm{N}$ & $\%$ & $\mathbf{N}$ & $\%$ & & \\
\hline \multicolumn{2}{|c|}{ BMI (kg/m 2) } & $\begin{array}{l}26.1 \\
(3.1)\end{array}$ & & $\begin{array}{l}29.3 \\
(3.7)\end{array}$ & & $\begin{array}{c}1.31 \\
(1.20-1.44)\end{array}$ & $<0.001$ \\
\hline \multirow[t]{2}{*}{$\begin{array}{l}\text { BMI } \\
\left(\mathrm{kg} / \mathrm{m}^{2}\right)\end{array}$} & $\begin{array}{l}\text { overwe } \\
\text { ight }\end{array}$ & 6 & 85.7 & 1 & 14.3 & $\begin{array}{c}3.97 \\
(0.47-33.54)\end{array}$ & 0.205 \\
\hline & obese & 136 & 60.2 & 90 & 39.8 & & \\
\hline \multicolumn{2}{|c|}{ WtH ratio } & $\begin{array}{c}0.61 \\
(0.06)\end{array}$ & & $\begin{array}{c}0.63 \\
(0,04 \\
)\end{array}$ & & $\begin{array}{c}1.10 \\
(1.02-1.18)\end{array}$ & 0.013 \\
\hline \multicolumn{2}{|l|}{$\%$ fat } & $\begin{array}{l}35.5 \\
(4.7)\end{array}$ & & $\begin{array}{c}38.8 \\
(0.05 \\
)\end{array}$ & & $\begin{array}{c}1.14 \\
(1.05-1.25)\end{array}$ & 0.003 \\
\hline W. C. & & $\begin{array}{c}85.6 \\
(10,4)\end{array}$ & & $\begin{array}{l}94.7 \\
(9,6)\end{array}$ & & $\begin{array}{c}1.10 \\
(1.05-1.14)\end{array}$ & $<0.001$ \\
\hline \multirow[t]{2}{*}{$\begin{array}{l}\text { F. Hy of } \\
\text { obesity }\end{array}$} & no & 53 & 54.1 & 45 & 45.9 & $\begin{array}{c}0.58 \\
(0.34-1.00)\end{array}$ & 0.051 \\
\hline & yes & 85 & 66.9 & 42 & 33.1 & & \\
\hline \multirow[t]{2}{*}{ A. $\mathrm{N}$. } & no & 120 & 64.5 & 66 & 35.5 & $\begin{array}{c}2.42 \\
(1.23-4.79)\end{array}$ & 0.011 \\
\hline & yes & 18 & 42.9 & 24 & 57.1 & & \\
\hline \multirow[t]{2}{*}{$\begin{array}{l}\text { Wt/Ht } \\
\text { ratio }\end{array}$} & $<0.5$ & 2 & 66.7 & 1 & 33.3 & $\begin{array}{c}1.62 \\
(0.14-18.27) \\
\end{array}$ & 0.698 \\
\hline & $>0.5$ & 73 & 55.3 & 59 & 44.7 & & \\
\hline
\end{tabular}

\begin{tabular}{|c|c|c|c|c|c|c|c|}
\hline & \multicolumn{4}{|c|}{ METABOLIC SYNDROME } & \multirow{3}{*}{$\begin{array}{c}\text { OR } \\
(95 \% \mathrm{Cl})\end{array}$} & \multirow{3}{*}{$\mathbf{P}$} \\
\hline & & \multicolumn{2}{|c|}{ No } & \multicolumn{2}{|c|}{ YES } & & \\
\hline & & $\mathbf{N}$ & $\%$ & $\mathbf{N}$ & $\%$ & & \\
\hline \multicolumn{2}{|l|}{ BMI (kg/m $\left.{ }^{2}\right)$} & \begin{tabular}{|c|}
26,1 \\
)
\end{tabular} & & $\begin{array}{l}28,4 \\
(3.9)\end{array}$ & & $\begin{array}{c}1.16 \\
(1.06-1.27)\end{array}$ & 0.001 \\
\hline \multirow[t]{2}{*}{ BMI $\left(\mathrm{kg} / \mathrm{m}^{2}\right)$} & $\begin{array}{l}\text { overwei } \\
\text { ght }\end{array}$ & 17 & 100.0 & 0 & 0,0 & - & \\
\hline & obese & 226 & 86.3 & 36 & 13.7 & & \\
\hline \multicolumn{2}{|l|}{ Wt/Ht ratio } & $\begin{array}{l}0.61 \\
(0.05)\end{array}$ & & $\begin{array}{l}0.63 \\
(0.05)\end{array}$ & & $\begin{array}{c}1.07 \\
(0.99-1.16)\end{array}$ & 0.100 \\
\hline \multicolumn{2}{|l|}{$\%$ Fat } & $\begin{array}{l}35,8 \\
(5.6)\end{array}$ & & $\begin{array}{r}36,7 \\
(3.6)\end{array}$ & & $\begin{array}{c}1.03 \\
(0.95-1.21)\end{array}$ & 0.462 \\
\hline W. C. & & $\begin{array}{l}87.5 \\
(10.1)\end{array}$ & & $\begin{array}{l}91,89 \\
(10.2)\end{array}$ & & $\begin{array}{c}1.05 \\
(1.00-1.09)\end{array}$ & 0.030 \\
\hline \multirow[t]{2}{*}{$\begin{array}{l}\text { Family hy } \\
\text { of obesity }\end{array}$} & no & 108 & 87.1 & 16 & 12.9 & $\begin{array}{c}1.06 \\
(0.53-2.5) \\
\end{array}$ & 0.865 \\
\hline & yes & 127 & 86.4 & 20 & 13.6 & & \\
\hline \multirow[t]{2}{*}{ A. $\mathrm{N}$. } & no & 206 & 88.4 & 27 & 11.6 & $\begin{array}{c}1.91 \\
(0.80-4.56)\end{array}$ & 0.147 \\
\hline & yes & 32 & 80.0 & 8 & 20.0 & & \\
\hline \multirow[t]{2}{*}{ Wt/ Ht ratio } & $<0.5$ & 2 & 66.7 & 1 & 33.3 & $\begin{array}{c}0.49 \\
(0,04-5.60)\end{array}$ & 0.568 \\
\hline & $>0.5$ & 130 & 80.2 & 32 & 19.8 & & \\
\hline
\end{tabular}

